\title{
ON NONLINEAR MODELS OF MARKETS WITH FINITE LIQUIDITY: SOME CAUTIONARY NOTES
}

BY KRISTOFFER J. GLOVER ${ }^{A, B *}$, PETER W. DUCK ${ }^{A, C}$ AND DAVID P. NEWTON ${ }^{D}$ $A$ SCHOOL OF MATHEMATICS, UNIVERSITY OF MANCHESTER, MANCHESTER, UK

$B$ PRESENT ADDRESS: SCHOOL OF FINANCE AND ECONOMICS, UNIVERSITY OF TECHNOLOGY, SYDNEY, AUSTRALIA

C CORRESPONDING AUTHOR. TEL:+44-161-275-5831; EMAIL:DUCK@MA.MAN.AC.UK

$D$ NOTTINGHAM UNIVERSITY BUSINESS SCHOOL, NOTTINGHAM, UK

\begin{abstract}
The recent financial crisis and related liquidity issues has illuminated an urgent need for a better understanding of the effects of limited liquidity on all aspects of the financial system. This paper considers such effects on the Black-ScholesMerton financial model, which for the most part result in highly nonlinear partial differential equations (PDEs). We investigate in detail a model studied by Schönbucher and Wilmott (2000) which incorporates the price impact of option hedging strategies. First, we consider a first-order feedback model, which leads to the exceptional case of a linear PDE. Numerical results, and more particularly an asymptotic approach close to option expiry, reveal subtle differences from the Black-Scholes-Merton model. Second, we go on to consider a full-feedback model in which price impact is fully incorporated into the model. Here, standard numerical techniques lead to spurious results in even the simplest cases. An asymptotic approach, valid close to expiry, is mounted, and a robust numerical procedure, valid for all times, is developed, revealing two distinct classes of behavior. The first may be attributed to the infinite second derivative associated with standard option payoff conditions, for which it is necessary to admit solutions with discontinuous first derivatives; perhaps even more disturbingly, negative option values are a frequent occurrence. The second failure (applicable to smoothed payoff functions) is caused by a singularity in the coefficient of the diffusion term in the option-pricing equation. Our conclusion is that several classes of model in the literature involving permanent price impact irretrievably break down (i.e. there is insufficient 'financial modeling' in the pricing equation). Our analysis should provide the necessary information to avoid such pitfalls in the future.
\end{abstract}

Key words. option valuation, numerical techniques, nonlinear finance, illiquid markets, price impact, market feedback, asymptotic analysis

AMS subject classifications. 62P05, 91B26, 91B28

1. Introduction. Since the definitive papers of Black and Scholes (1973) and Merton (1973), much of the work undertaken in mathematical finance has been aimed at relaxing a number of the underlying assumptions. One of the more subtle was that the market in the underlying was infinitely (or perfectly) liquid, such that trading had no impact on the price of the underlying. If we relax this assumption, then we see some rather interesting and possibly counterintuitive behaviors. As will be shown later, this is partly due to the fact that any model incorporating such a feature will inevitably lead to nonlinear behavior (feedback). In particular, we shall be concerned

*EPSRC FUNDING GRATEFULLY ACKNOWLEDGED. THE AUTHORS WISH TO THANK PETER BANK, LJUDMILA BORDAG, ERIK EKSTRÖM, ANDREW HAZEL, PAUL JOHNSON AND GORAN PESKIR FOR THEIR ADVICE AND ENLIGHTENING DISCUSSIONS. THE HELPFUL SUGGESTIONS OF THE ANONYMOUS REFEREES ARE ALSO GRATEFULLY ACKNOWLEDGED. 
with nonlinear partial differential equations (PDEs) arising from the study of finitely liquid markets and to providing insights into the governing PDEs and their nonlinearities. Work that has led to this class of PDEs in finance to date includes Whalley and Wilmott (1993) in relation to transaction costs, which was one of the first nonlinear PDEs to arise in the field of mathematical finance. In addition, there is the so called Black-Scholes-Barrenblatt equation introduced by Avellaneda et al. (1995) in the study of uncertain volatility models. These models involve optimization over all possible values of volatility, and as a result are also highly nonlinear.

The models we consider involve the price impact due to a large trader who is able to move the price by his/her actions. This impact can be considered permanent since it will affect the underlying price dynamics for other market participants. In the presence of such price impact, it is not clear whether an option is still perfectly replicable or not (i.e. if the market is complete); hence it is no longer straightforward how to derive option prices from the prices of the underlying asset. Frey and Stremme (1997) showed that if a hedging strategy is used that does not take into account the feedback effect (which we term first-order feedback), then it is not possible to replicate perfectly an option, and hence there is still risk associated with such a hedging strategy. They did show, however, that increasing heterogeneity of the distribution of hedged contracts reduces both the level and price sensitivity of this un-hedged risk. Frey $(1998,2000)$ then showed that if feedback is taken into account in a more general hedging strategy (which we term full feedback), then it is possible to replicate an option perfectly. In the discrete-time framework of Jarrow (1994), the question as to whether options could be perfectly replicated in a finitely liquid market reduces to solving (recursively) a finite number of equations. In the continuous time framework of Frey (1998) this can be characterized more succinctly as the solution to a nonlinear PDE, to which Frey (1998) gave existence and uniqueness results. These results, however, place a heavy restriction on the amount of market illiquidity that the model allows and rely on the terminal payoff to be sufficiently smooth, both of which can be seen as undesirable restrictions. Frey and Patie (2002) extended the work of Frey (2000) with an asset dependent liquidity parameter which attempts to incorporate so called liquidity drops, i.e. that market liquidity drops if the stock price drops, the aim being to reproduce the volatility smile.

Other continuous time models similar to Frey (1998) that incorporate permanent price impact include Schönbucher and Wilmott (2000), who used a market microstructure equilibrium model to derive a modified stochastic process under the influence of price impact. The PDEs derived by these latter authors correspond to those derived in section 2 of the present study. Sircar and Papanicolaou (1998) derived a slightly different nonlinear PDE that depends on the exogenous income process of reference traders and the relative size of the large trader. Platen and Schweizer (1998) proposed a similar microstructure model as an endogenous explanation of the volatility smile and its skewness. Lyukov (2004) extended this model with more realistic assumptions about market equilibrium conditions and also obtained a very similar nonlinear PDE to that derived in section 2. Another 'tweak' of these models was made by Liu and Yong (2005) who attempted to regularize the PDE close to expiry. A related, but crucially different, class of models was developed by Cetin et al. (2004, 2006) and Cetin and Rogers (2007) amongst others. These models can be considered as reduced form models, since they do not allow for any permanent price impact of trades. These models eliminate the feedback effects discussed above, however the assumption of only transitory price impact does not appear to be econometrically justifiable (see, for ex- 
ample, Section 3.3.2 in Madhavan, 2000). For this reason we do not consider such models here.

The present study is primarily concerned with the behavior of a canonical form of nonlinear PDEs encompassing the work of Schönbucher and Wilmott (2000), Frey (1998) and Liu and Yong (2005) amongst others. Motivated by the various criticisms of these models, some interesting and undesirable properties are outlined. Our eventual aim is to warn against breakdown in solution of models across the area of liquidity/derivatives modeling, showing how this arises, where and how it can be tackled, shedding some light on to how these models may be modified and thereby improved. The layout of this paper is as follows: section 2 provides a derivation of the relevant pricing PDEs and transposes these results into a market with finite liquidity. In section 3 we investigate the behavior of a first-order feedback model and in section 4 we go on to consider a fully nonlinear, infinite feedback model. Here we suggest a structure to describe the behavior of the model close to expiry, which provides some interesting consequences and insights for early exercise options and serves to guide the numerical work presented in this section. In section 5 we investigate how even payoffs which have been smoothed to avoid some of the aforementioned difficulties may themselves exhibit (alternative) singular pricing behaviors and we provide conditions under which we should expect these to occur. Then in section 6 we briefly discuss the valuation of perpetual American options in the present framework. Finally we summarize our conclusions in section 7 .

2. The model. In order to provide a brief derivation of the primary governing PDEs considered in this paper, we present the following intuitive arguments, which are similar to those employed in Lipton (2001) and Liu and Yong (2005). Given a general stochastic process, which we shall assume to be geometric Brownian motion (but can be generalized to any stochastic process)

$$
d S=\mu S d t+\sigma S d W_{t}
$$

where $S$ is the price of the underlying, $\mu$ and $\sigma$ are the (constant) drift and volatility respectively and $W_{t}$ is a standardized Brownian motion. It is possible to add a forcing term, $f(S, t)$, to the process, i.e.

$$
d S=\mu S d t+\sigma S d W_{t}+\lambda(S, t) d f
$$

where $\lambda(S, t)$ is an arbitrary function. Note that at this stage no assumptions are being made regarding the form of the functions $\lambda(S, t)$ and $f(S, t)$, and particular financial interpretations can conveniently be postponed until certain manipulations are complete.

Since $f(S, t)$ is a function of $S$ and $t$ only, it is possible to incorporate the additional contribution to the price dynamics into the drift and volatility coefficients $\mu$ and $\sigma$. We commence by applying Itô's formula to the function $f(S, t)$, to obtain

$$
d f=\frac{\partial f}{\partial t} d t+\frac{\partial f}{\partial S} d S+\frac{1}{2} \frac{\partial^{2} f}{\partial S^{2}}(d S)^{2}+\ldots
$$

which substituting into (2.2), gives to leading order

$$
\left(1-\lambda \frac{\partial f}{\partial S}\right) d S=\left(\mu S+\lambda \frac{\partial f}{\partial t}\right) d t+\frac{\lambda}{2} \frac{\partial^{2} f}{\partial S^{2}}(d S)^{2}+\sigma S d W_{t}
$$


In order to proceed further we require an expression for $(d S)^{2}$, which can be obtained by simply squaring equation (2.3) to yield, as $d t \rightarrow 0$

$$
(d S)^{2}=\frac{\sigma^{2} S^{2} d t}{\left(1-\lambda \frac{\partial f}{\partial S}\right)^{2}}+o(d t)
$$

where we have used the standard (heuristic) condition that $\left(d W_{t}\right)^{2} \rightarrow d t$ as $d t \rightarrow 0$. Substituting (2.4) into (2.3), and with a little rearranging, we arrive at the following stochastic process, analogous to (2.1):

$$
d S=\hat{\mu}(S, t) d t+\hat{\sigma}(S, t) d W_{t},
$$

where

$$
\hat{\mu}(S, t)=\frac{1}{1-\lambda \frac{\partial f}{\partial S}}\left[\mu S+\lambda\left(\frac{\partial f}{\partial t}+\frac{1}{2} \hat{\sigma}^{2} \frac{\partial^{2} f}{\partial S^{2}}\right)\right], \quad \hat{\sigma}(S, t)=\frac{\sigma S}{1-\lambda \frac{\partial f}{\partial S}} .
$$

We can interpret the function $f(S, t)$ as a forcing mechanism on an underlying stochastic process which results in the process (2.1) being modified to the process (2.5).

We now turn our attention to option pricing under this modified stochastic process. To do this we will use the well-known Generalized Black-Scholes equation (for a detailed derivation see, for example, Duffie, 1996), which leads to the following pricing PDE for the modified stochastic process incorporating the forcing term

$$
\frac{\partial V}{\partial t}+\frac{1}{2} \frac{\sigma^{2} S^{2}}{\left(1-\lambda \frac{\partial f}{\partial S}\right)^{2}} \frac{\partial^{2} V}{\partial S^{2}}+r S \frac{\partial V}{\partial S}-r V=0 .
$$

Note that, consistent with standard Black-Scholes arguments, the drift of the modified process $\hat{\mu}(S, t)$ does not appear in the option pricing PDE.

Thus far we have been deliberately vague about the financial interpretation of the forcing term in (2.1). In the context of markets with finite liquidity, we can define $f(S, t)$ to be the number of extra shares traded due to some deterministic hedging strategy and $\lambda(S, t)$ as some function dependent on how we choose to model the form of price impact. Markets are not complete to traders who do not have the opportunity to trade continuously. Large institutions can trade close to continuously and so options in a complete market provide no extra trading opportunities to them. For small traders however, options open up new trading possibilities, resulting in the large institution selling the options to the small traders and then hedging the risk by replicating the option. This leads to a high demand for these replicating strategies by large financial institutions and it is not, therefore, unreasonable to assume that a trading strategy that could impact the price significantly (due to the large trading volumes) is that of delta hedging. In this case the trading strategy $f$, in equation (2.7) should be set to an option delta, defined as $\Delta=\partial V / \partial S$, based on some form of option $V^{*}$, i.e.

$$
f=\frac{\partial V^{*}}{\partial S}
$$

This leads to an interesting question about which strategy the hedgers are assumed to follow. A naive strategy would be if $V^{*}$ were the Black-Scholes value $V^{\mathrm{BS}}$ and thus 
distinct from the solution $V$ of equation (2.7). This leads to the linear PDE

$$
\frac{\partial V}{\partial t}+\frac{1}{2} \frac{\sigma^{2} S^{2}}{\left(1-\lambda \frac{\partial^{2} V^{\mathrm{BS}}}{\partial S^{2}}\right)^{2}} \frac{\partial^{2} V}{\partial S^{2}}+r S \frac{\partial V}{\partial S}-r V=0
$$

This case we call first-order feedback, which is analyzed in detail in section 3 . The Black-Scholes case is recovered by setting $\lambda=0$ in (2.9). Another (more interesting and challenging) case is when the hedger is assumed to be aware of the feedback effect and so would change the hedging strategy accordingly. We shall call this case full feedback, which corresponds to the case when $V^{*} \equiv V$, i.e. the trading strategy adopted has to be found as part of the problem. This leads to the fully nonlinear PDE,

$$
\frac{\partial V}{\partial t}+\frac{1}{2} \frac{\sigma^{2} S^{2}}{\left(1-\lambda \frac{\partial^{2} V}{\partial S^{2}}\right)^{2}} \frac{\partial^{2} V}{\partial S^{2}}+r S \frac{\partial V}{\partial S}-r V=0,
$$

which is dealt with in section 4 .

Equation (2.10) has appeared in the literature several times with differing forms of the function $\lambda(S, t)$ according to the modeling assumptions. The reader is directed to the original papers for more on these assumptions and on the underlying economic rationale. The simplest case occurs in Schönbucher and Wilmott (2000), who have $\lambda(S, t)$ constant and a dimensionless measure of the liquidity of the market. Frey (1998) has the similar form $\lambda(S, t)=\hat{\lambda} S$ where $\hat{\lambda} \in \mathbb{R}^{+}$is again some measure of the liquidity of the market. Another form for $\lambda(S, t)$ can be found in Liu and Yong (2005) in which $\lambda(S, t)=\hat{\lambda}\left(1-e^{-\beta(T-t)}\right)$ where $\hat{\lambda}$ is a constant price impact coefficient, $T-t$ is time to expiry and $\beta$ a decay coefficient. As far as we can ascertain there is little financial justification for this, and it appears to be introduced for numerical expediency to avoid difficulties associated with the growing second derivative of the option (the gamma) as expiration approaches. In what follows it is assumed for the most part that $\lambda(S, t)$ is a constant, analogous with the work of Schönbucher and Wilmott (2000), although a good deal of the (small-time) analysis presented here is quite widely applicable to other models.

In the next section we consider the case of first-order feedback and show how the illiquidity, even in this case, has a significant effect on the option replication price, especially as we approach expiry.

3. First-order feedback model. As a starting point to investigating how liquidity can affect the option value, we assume that a single option is to be hedged and furthermore that the hedger holds the number of stocks dictated by the analytical Black-Scholes delta, rather than the delta from the modified option price. This leads to the linear PDE (2.9) which is somewhat easier to solve than the full-feedback problem, but still has important and interesting differences from the classical BlackScholes PDE. This idea of first-order feedback leading to a modified, but still linear, Black-Scholes PDE also appeared in Schönbucher and Wilmott (2000), but under a different guise. They call the solution to the PDE (2.9) the price taker's price. In an illiquid market influenced by a large trader (or by an equivalent large group of small traders following the same trading strategy) a small trader can trade any number of shares, on a small scale, without affecting the price. Hence equation (2.9) models the replicating cost of an option for such small traders; for these traders only, the market appears liquid. 


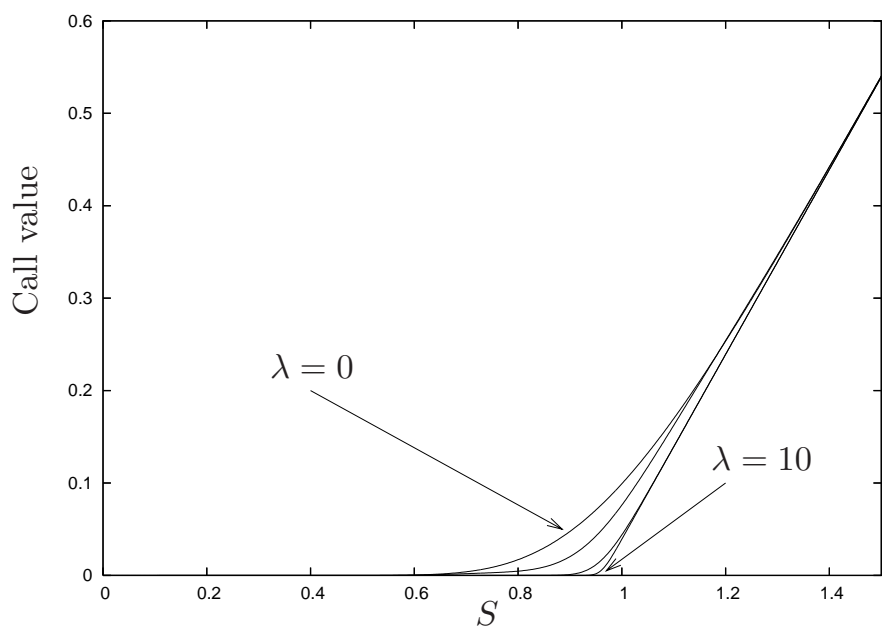

FIG. 3.1. Value of European call options with first-order feedback ( $T=1, r=0.04, \sigma=0.2$, $X=1)$ for $\lambda=0,1,2,5,10$; the variation with $\lambda$ is monotonic.

Figure 3.1 shows numerical results from the solution of equation (2.9) (obtained using a Crank-Nicolson procedure) for European call options (all with time to maturity, $T=1$ year, risk-free rate, $r=0.04$, volatility, $\sigma=0.2$, and exercise price, $X=1)$ for $\lambda=0,1,2,5,10$. Here, the standard call expiry payoff at $t=T$ has been implemented, i.e.

$$
C(S, T)=(S-X)^{+}
$$

The result with $\lambda=0$ is, as noted above, the classic Black-Scholes result. As $\lambda$ is increased, the option value is apparently eroded monotonically towards the amount by which the contract is currently in the money or, if out of the money, zero. Corresponding results for put options (using the same parameters as for figure 3.1), with the standard put payoff condition

$$
P(S, T)=(X-S)^{+}
$$

are presented in figure 3.2 , and these, too, strongly point to a monotonic asymptote on to the payoff function (for fixed $T$ ) as the liquidity parameter $\lambda$ increases. Interestingly, although this convergence to the payoff function appears monotonic, it is not. The behavior of the option price as $\lambda$ varies depends on the subtle interplay of $V_{S S}^{\mathrm{BS}}$ and $\lambda$ at any given $S$ and $\tau$. This behavior was investigated further in Glover (2008) and in the interests of brevity the interested reader is directed there.

Although the illiquid results above appear to be rather qualitatively similar to the liquid $(\lambda=0)$ results, a more detailed analysis (applicable for times close to expiry) follows, and this highlights some subtle, but important differences.

3.1. Analysis close to expiry: European options. Consider the behavior of the option value close to expiry. This is generally the most critical and intricate period for option pricing models and offers us some insight into the valuation dynamics, shedding more light on the value of options as the parameter $\lambda$ is increased.

We now consider the regime close to expiry and also close to the strike price (i.e. at the money), which for most option valuation problems is the most intricate 


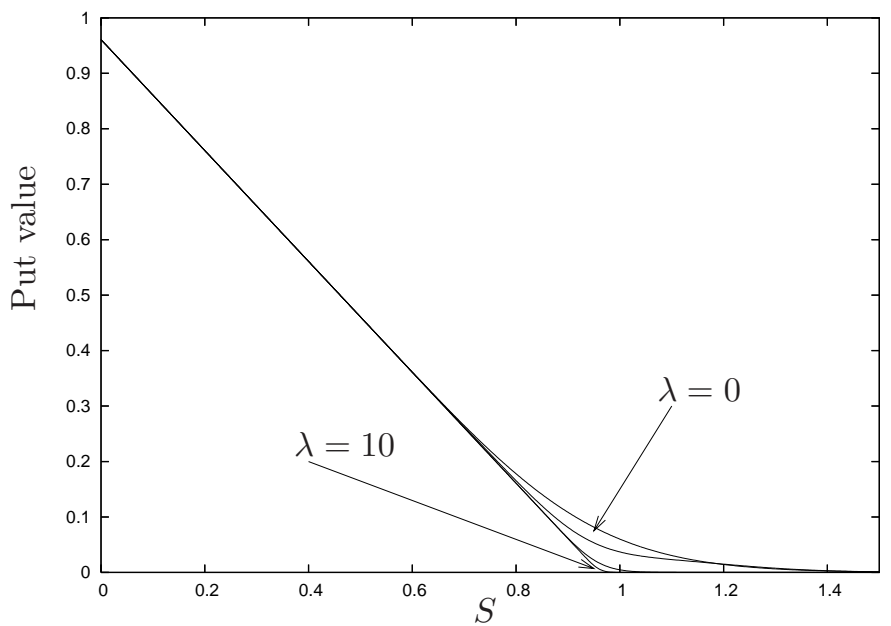

FIG. 3.2. Value of European put options with first-order feedback ( $T=1, r=0.04, \sigma=0.2$, $X=1)$ for $\lambda=0,1,2,5,10$; the variation with $\lambda$ is monotonic.

in solution space. We first consider the behavior of the (standard) Black-Scholes equation in this regime. To obtain this, we use the well known result that as the time to expiry $\tau=T-t \rightarrow 0$ locally the solution takes the form

$$
V^{\mathrm{BS}}=\tau^{\frac{1}{2}} f(\eta)+O(\tau),
$$

where

$$
\eta=\frac{S-X}{\tau^{\frac{1}{2}}},
$$

which can be verified a posteriori. It can be shown (see for example Wilmott et al., 1995) that the solution when $\eta=O(1)$ for a put is

$$
f^{\mathrm{P}}(\eta)=\eta\left[\Phi\left(\frac{\eta}{\Lambda}\right)-1\right]+\frac{\Lambda}{\sqrt{2 \pi}} e^{-\frac{1}{2}\left(\frac{\eta}{\Lambda}\right)^{2}},
$$

and likewise for a call

$$
f^{\mathrm{C}}(\eta)=\eta \Phi\left(\frac{\eta}{\Lambda}\right)+\frac{\Lambda}{\sqrt{2 \pi}} e^{-\frac{1}{2}\left(\frac{\eta}{\Lambda}\right)^{2}}
$$

where $\Lambda=\sigma X$ and $\Phi(\cdot)$ is the standard normal cumulative distribution function defined as

$$
\Phi(x)=\frac{1}{\sqrt{2 \pi}} \int_{-\infty}^{x} e^{-\frac{1}{2} y^{2}} d y .
$$

We can now proceed to incorporate this into the first-order feedback illiquid problem (2.9). To investigate the small $\tau$ behavior of this equation, analogous to the above local analysis, we seek a solution of the form

$$
V=\tau g(\xi), \quad \text { where } \quad \xi=\frac{S-X}{\tau},
$$


forms which can also be verified a posteriori. Note that the scaling here implies a region $O(\tau)$ in asset space $S$, close to the strike price, that is somewhat smaller than the classical Black-Scholes model (which is $O\left(\tau^{\frac{1}{2}}\right)$ as $\tau \rightarrow 0$ - see (3.4)); this is clearly an important difference from the standard Black-Scholes model behavior close to expiry. Note that on the relatively short $\xi=O(1)$ scale, using (3.3), (3.5) and (3.6) it is entirely rational to write (for both puts and calls)

$$
V_{S S}^{\mathrm{BS}}=\frac{1}{\sigma X \sqrt{2 \pi \tau}} .
$$

Then substitution of (3.7) and (3.8) into (2.9) yields to leading order

$$
\frac{\pi \sigma^{4} X^{4}}{\lambda^{2}} g_{\xi \xi}+(\xi+r X) g_{\xi}-g=0
$$

with the scaled boundary conditions becoming, for a put:

$$
g \rightarrow 0 \text { as } \xi \rightarrow \infty, \quad g \rightarrow-(\xi+r X) \text { as } \xi \rightarrow-\infty
$$

and for a call:

$$
g \rightarrow \xi+r X \text { as } \xi \rightarrow \infty, \quad g \rightarrow 0 \text { as } \xi \rightarrow-\infty
$$

Equation (3.9) can be solved analytically. For a put, the solution is

$$
g^{\mathrm{P}}(\xi)=(\xi+r X)\left[\Phi\left(\frac{\xi+r X}{\kappa}\right)-1\right]+\frac{\kappa}{\sqrt{2 \pi}} e^{-\frac{1}{2}\left(\frac{\xi+r X}{\kappa}\right)^{2}},
$$

where $\kappa=\frac{\sqrt{\pi} \sigma^{2} X^{2}}{\lambda}$, and for a call

$$
g^{\mathrm{C}}(\xi)=(\xi+r X) \Phi\left(\frac{\xi+r X}{\kappa}\right)+\frac{\kappa}{\sqrt{2 \pi}} e^{-\frac{1}{2}\left(\frac{\xi+r X}{\kappa}\right)^{2}} .
$$

Note that increasing illiquidity $(\lambda \rightarrow \infty)$ implies $\kappa \rightarrow 0$ and this in turn indicates that (3.10) and (3.11) become increasing focused about $\xi=-r X$, i.e. $S=X(1-r \tau)$, taking on the payoff form away from this point, consistent with our observations above regarding figures 3.1 and 3.2. Also it can be seen that call values always lie above the payoff curve $\left(g^{\mathrm{C}}-(\xi)^{+}>0\right.$ for all $\left.\xi\right)$. As with the classical Black-Scholes result for European calls, in the present case it is never optimal to exercise calls early. However it is possible to have $g^{\mathrm{P}}-(-\xi)^{+}<0$ (i.e. $V-$ payoff $<0$ ) for certain ranges of $\xi$, which opens up the potential for the optimal early exercise (on the $\xi$ scale), and so a consideration of this possibility is considered next.

3.2. Analysis close to expiry: American options. The remarks above naturally beg the question as to the value of put options on finitely liquid underlyings if early exercise is permitted. In the context of first-order feedback, the most consistent model has the delta in (2.8) computed using the liquid $(\lambda=0)$ American put value $V_{\mathrm{AM}}^{\mathrm{BS}}$, which does permit early exercise on the $\xi=\mathcal{O}(1)$ scale; note that the free boundary (optimal exercise price) of the illiquid put option, $V$, need not necessarily be the same as the free boundary of the liquid option $V_{\mathrm{AM}}^{\mathrm{BS}}$. Figure 3.3 shows results for the American put with the same financial parameters as for the earlier European options, obtained via a standard Projected Successive Over Relaxation (PSOR) iterative procedure. At each iteration, first $V_{\mathrm{AM}}^{\mathrm{BS}}=\max \left\{\hat{V}_{\mathrm{AM}}^{\mathrm{BS}}, X-S\right\}$ was taken, where 


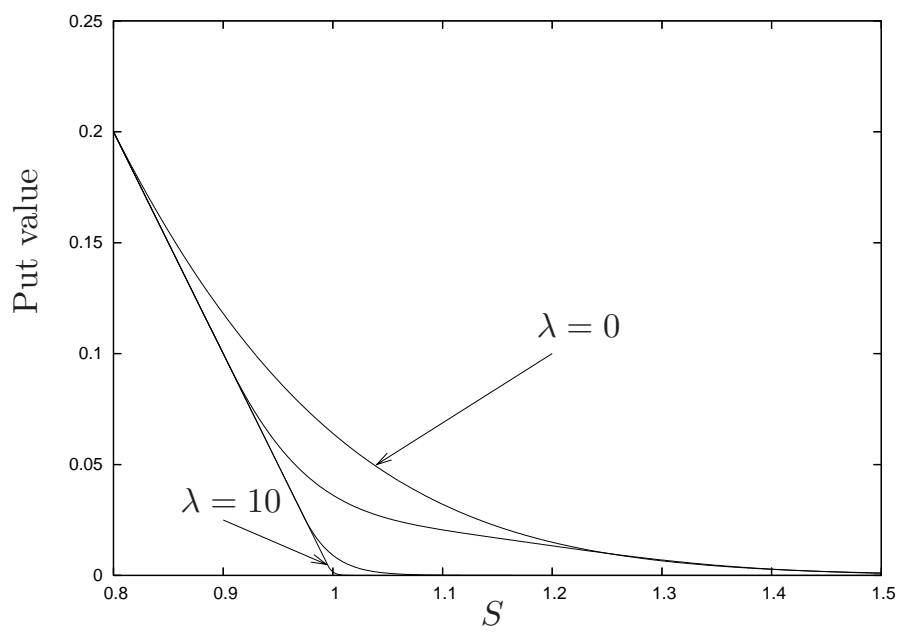

Fig. 3.3. Value of American put options, $T=1, r=0.04, \sigma=0.2, X=1$ and for $\lambda=0,1$, 2, 5, 10; the variation with $\lambda$ is monotonic.

$\hat{V}_{\mathrm{AM}}^{\mathrm{BS}}$ is the solution to the Black-Scholes equation subject to the payoff conditions (3.2). Then $V$ was determined by taking $\max \left\{\hat{V}_{\mathrm{AM}}, X-S\right\}$ where $\hat{V}_{\mathrm{AM}}$ is computed from (2.9), also subject to (3.2). Again we see the 'collapse' of the option value on to the payoff as the liquidity parameter $\lambda$ is increased (which implies the location of the free boundary always moves towards the exercise price as $\lambda$ increases). However, although the results appear to be qualitatively similar to the $\lambda=0$ case, there are subtle differences, as we shall now show.

Analysis of the liquid $(\lambda=0)$ American put option close to expiry leads to a somewhat complicated structure, as detailed by Kuske and Keller (1998). Here, the $\eta$ scale defined in (3.4) can be shown to fail to capture the free (exercise) boundary. Instead, the free boundary is located at a somewhat larger distance $(O(\sqrt{-\tau \log \tau}))$ from the exercise price (with a significant price variation in a region $O(\sqrt{-\tau / \log \tau})$ of this exercise boundary). It was shown by Widdicks (2002) that as $\tau \rightarrow 0$, on the $\eta=O(1)$ scale, the solution of the liquid $(\lambda=0)$ American option takes the same form as that of its European counterpart, i.e. (3.5). Therefore, it is entirely self consistent to use this form and, indeed, the European gamma for the American case when $\eta=O(1)$ or smaller, which is relatively distant from the free boundary. However, recall that for the case when $\lambda \neq 0$, with $\xi=O(1)$ (see (3.7)) we have clear indications of the possibility of early exercise for the illiquid put. Therefore, the American problem in this case reduces to the solution of (3.9), subject to

$$
\begin{aligned}
& g \rightarrow 0 \quad \text { as } \quad \xi \rightarrow \infty \\
& g=-\xi \quad \text { and } \quad g_{\xi}=-1 \quad \text { on } \quad \xi=\xi_{s},
\end{aligned}
$$

where we have used the usual smooth pasting conditions (continuity of the option value and its derivative) and $\xi_{s}$ denotes the location of the free boundary (on the $\xi$ scale). It is possible to reduce the above problem to a transcendental equation for $\xi_{s}$ namely,

$$
\Phi\left(\frac{\lambda\left(\xi_{s}+r X\right)}{\sqrt{\pi} \sigma^{2} X^{2}}\right)=1-\frac{\sigma^{2} X}{\sqrt{2} \lambda r} e^{-\frac{\lambda^{2}}{2 \pi}\left(\frac{\xi_{s}+r X}{\sigma^{2} X^{2}}\right)^{2}}
$$




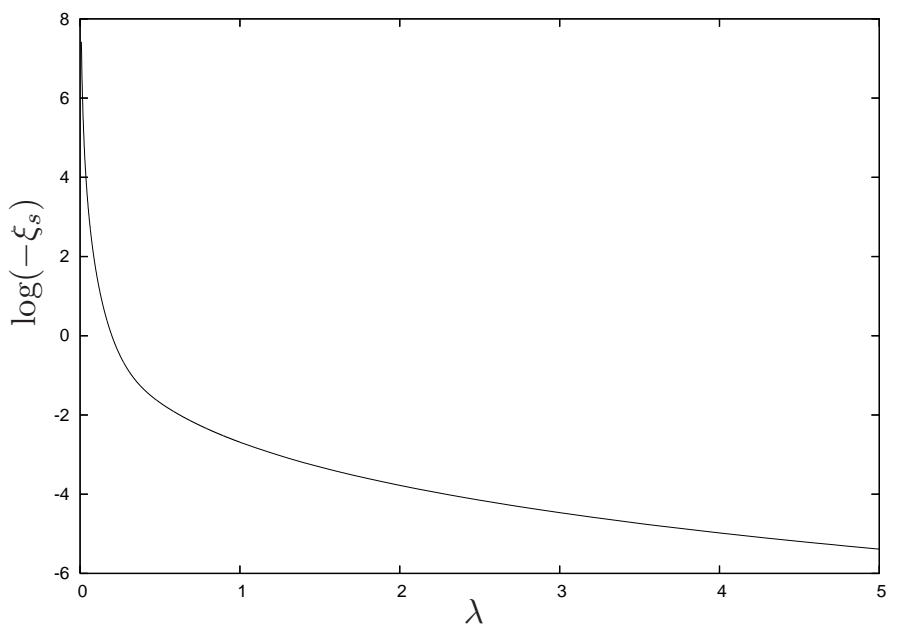

FIG. 3.4. First-order feedback put (with early exercise), location of free boundary (as $\tau \rightarrow 0$ ) with $\lambda, X=1, r=0.04, \sigma=0.2$.

but it is equally straightforward to solve the system (3.9), (3.12), (3.13) fully numerically and both procedures were adopted to check for consistency/accuracy.

Figure 3.4 shows the variation of the local free boundary $\xi_{s}$ (more particularly $\left.\log \left(-\xi_{s}\right)\right)$ with $\lambda$ for the financial parameters considered earlier, i.e. $r=0.04, \sigma=0.2$, $X=1$. The key point to note is that solutions of the system do exist, i.e. the short $S-X=O(\tau), \xi=O(1)$ scale captures the location of the free boundary with firstorder feedback, whilst as noted above, the liquid $(\lambda=0)$ case evolves on a relatively longer scale of $S-X=O(\sqrt{-\tau \log \tau})$; consistent with this as $\lambda \rightarrow 0$, we have that $\xi_{s} \rightarrow-\infty$. Further asymptotic analysis can describe this behavior, but is omitted in the interests of brevity. With this necessary background complete, we may proceed to investigate the full-feedback model.

4. Full-feedback model. We now turn our attention to the full feedback case, namely equation (2.10), where the trading strategy assumed to affect the price is not simply the Black-Scholes delta hedging strategy as discussed in the previous section, but rather is based on the 'actual' delta of the modified option price and, as a consequence the price impact is fully considered in the trading strategy. This corresponds to a situation where all market participants performing such hedging strategies are aware of the effect that their strategies have on the price. In this case the trading strategy has to be determined as part of the problem, resulting in nonlinearity. The full-feedback model described here is what Schönbucher and Wilmott (2000) call the paper value replication for the large trader. Liquidating the portfolio would change the price and, due to the negative slope of the demand curve, the realized value would, inevitably, be less than this paper value. However, numerous difficulties arise when liquidation strategies are incorporated into such dynamic hedging strategies. It is for this reason that the majority of models in the literature (including the present study) consider only the paper value or make the assumption that the option position is closed out using physical delivery to bypass any difficulties with the liquidation value. Also implicit in the model is the assumption of zero transaction costs (such as brokerage); a reasonable assumption for a large trader who can exploit economies of scale. 


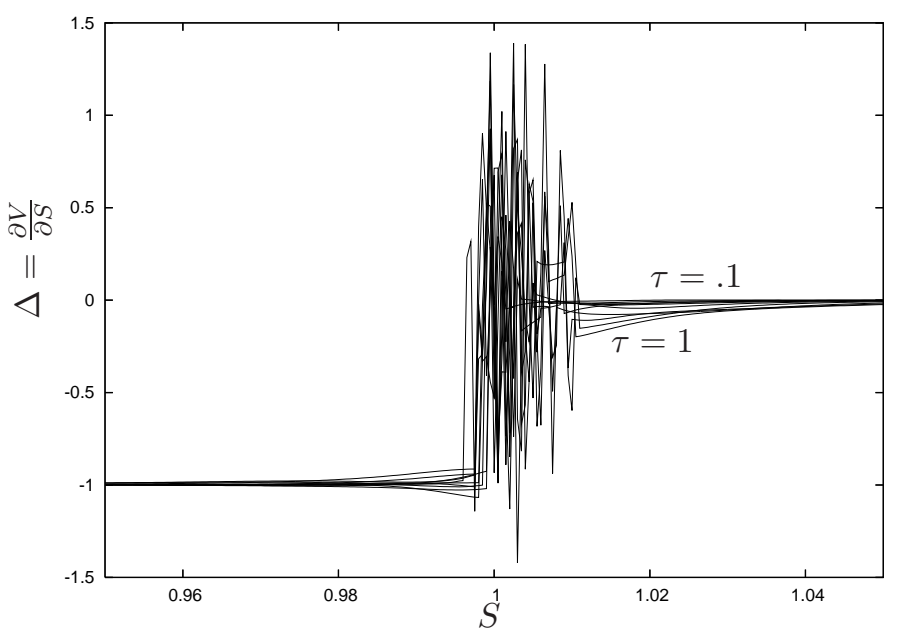

Fig. 4.1. Deltas for full-feedback (European) put, $X=1, r=0.04, \sigma=0.2, \lambda=0.1, T=1$.

Consider a numerical treatment of (2.10), subject to the put payoff condition (3.2). Figure 4.1 shows results obtained using a similar Crank-Nicolson scheme to that successfully employed on the first-order feedback model but, of course, incorporating iteration in order to treat properly the inherent nonlinearity in the problem. The results (for the delta) are clearly erroneous, even though they were obtained with a relatively fine grid (time-step $\Delta \tau$ of $10^{-3}$, grid-size of $5 \times 10^{-4}$ ); in addition, the output was found to be highly dependent on the choice of grid.

These difficulties are understandably side-stepped in published works, but a study of the causes will surely be helpful for the next phase of modeling in the field. In fact there are two problematic issues with regard to these difficulties, which are not unconnected. The first is linked to the inevitable infinite behavior of the gamma with standard payoff conditions, which even a cursory inspection of (2.10) suggests will be problematic; this is considered below. The second difficulty (again revealed by a cursory inspection of (2.10)) occurs if there is a zero in the denominator of the volatility term. A discussion of this issue will be deferred until section 5 .

4.1. Analysis close to expiry. As noted earlier, a thorough asymptotic analysis of the option valuation close to maturity $(\tau \rightarrow 0)$ can yield significant insight into the dynamics of the problem, and consequently this limit is studied next. For this we seek a local solution for the put value of the form (which can be justified a posteriori)

$$
V(S, \tau)=-\tau^{\frac{1}{2}} \eta \mathcal{H}(-\eta)+\tau \phi(\eta)+\ldots,
$$

where $\eta$ is defined in (3.4). $\mathcal{H}(\cdot)$ denotes the Heaviside function, which is necessary to 'mimic' the behavior of the payoff, close to expiry. Consequently, we have a different form for the valuation equation in two regions, one in $S>X$ (above the strike) and the other in $S<X$ (below the strike). Thus, although the option value is assumed to be continuous, clearly we are allowing for a discontinuous delta. Indeed, we sought solutions with a continuous delta, without success, and it is our assertion that such solutions do not exist for this problem. It should be noted that the above indicates the crucial regime is within a distance $O\left(\tau^{\frac{1}{2}}\right)$ of the exercise price as $\tau \rightarrow 0$ (a result determined through asymptotic analysis), similar to the $\lambda=0$ liquid options (as 
discussed in the previous section), which is rather broader than the scale appropriate for the first-order feedback options (which is $S-X=O(\tau)$ ).

The following equation then describes $\phi$ :

$$
\phi-\frac{\eta}{2} \phi_{\eta}-\frac{\sigma^{2} X^{2} \phi_{\eta \eta}}{2\left(1-\lambda \phi_{\eta \eta}\right)^{2}}+r X \mathcal{H}(-\eta)=0,
$$

with $\phi \rightarrow 0$ as $\eta \rightarrow \infty$ and $\phi \rightarrow-r X$ as $\eta \rightarrow-\infty$. At $\eta=0$, smooth pasting $\left(\phi, \phi_{\eta}\right.$ continuous) is appropriate. Sample results for a put option are shown in figure 4.2 (obtained via a standard shooting method). These results indicate that the option values all lie below the payoff (the repercussions of this will be discussed below). Note also the slower decay to the $|\eta| \rightarrow \infty$ asymptotes as the volatility increases due to the $O\left(\sigma^{2} X^{2}\right)$ scaling that emerges from (4.2) in these limits.

It is also straightforward to show (see Glover, 2008) that

$$
\phi^{\text {call }}(\eta)=\phi^{\text {put }}(-\eta)+r X,
$$

i.e., we can recover the local solution for calls from that of puts. The above symmetry relation can be shown to be simply a local manifestation of the global put-call parity relationship which holds for all time, even in this highly nonlinear case (provided early exercise is not permitted). This can be easily seen by direct substitution of the parity relationship $V^{\mathrm{P}}=V^{\mathrm{C}}-S+X e^{-r(T-t)}$ into equation (2.10).

The key observation in the above is the discontinuity in the delta at $\eta=0$ as indicated in (4.1), and it is the neglect of this that is undoubtedly responsible for the apparent spurious results observed in figure 4.1. Another point to be noted is that figure 4.2 indicates the possibility of negative put options values, a somewhat undesirable property (although (4.3) indicates this is not the case with calls).

Before a consideration of the problem for calculations for non-small values of $\tau$ (i.e. at times away from expiry), it turns out that yet another anomaly occurs, this time in the limit as $\sigma$ decreases (with other parameters held fixed). For values of $\sigma$ just below 0.15 (taking the other parameters used in figure 4.1, the numerical treatment applied to (4.2) failed, with the onset of negative roots in the computation.

To understand this, we rewrite (4.2) in the form of a quadratic in $\phi_{\eta \eta}$;

$$
2 \lambda^{2} \psi \phi_{\eta \eta}^{2}-\left(4 \lambda \psi+\sigma^{2} X^{2}\right) \phi_{\eta \eta}+2 \psi=0,
$$

where $\psi=\phi-\frac{\eta}{2} \phi_{\eta}+r X \mathcal{H}(-\eta)$. Using the quadratic formula we can write the 'solution' for $\phi_{\eta \eta}$ as

$$
\phi_{\eta \eta}=\left(\frac{1}{\lambda}+\frac{\sigma^{2} X^{2}}{4 \lambda^{2} \psi}\right)-\frac{\sigma^{2} X^{2}}{4 \lambda^{2} \psi}\left(1+\frac{8 \lambda \psi}{\sigma^{2} X^{2}}\right)^{\frac{1}{2}},
$$

where we have taken the negative root in order to satisfy the condition that $\phi_{\eta \eta} \rightarrow 0$ as $|\eta| \rightarrow \infty$. Indeed, this is the form that was taken as the basis of the numerical treatment used to obtain the results shown in figure 4.2, and inspection of the results indicated that difficulties arose if

$$
1+\frac{8 \lambda \psi}{\sigma^{2} X^{2}}<0
$$

Hence, we may expect this regime to arise for large values of the ratio $\lambda / \sigma^{2} X^{2}$, i.e. for sufficiently large $\lambda$, or sufficiently small $\sigma$. We shall return to a consideration of this regime in the following subsection, which concerns itself with the full problem. 


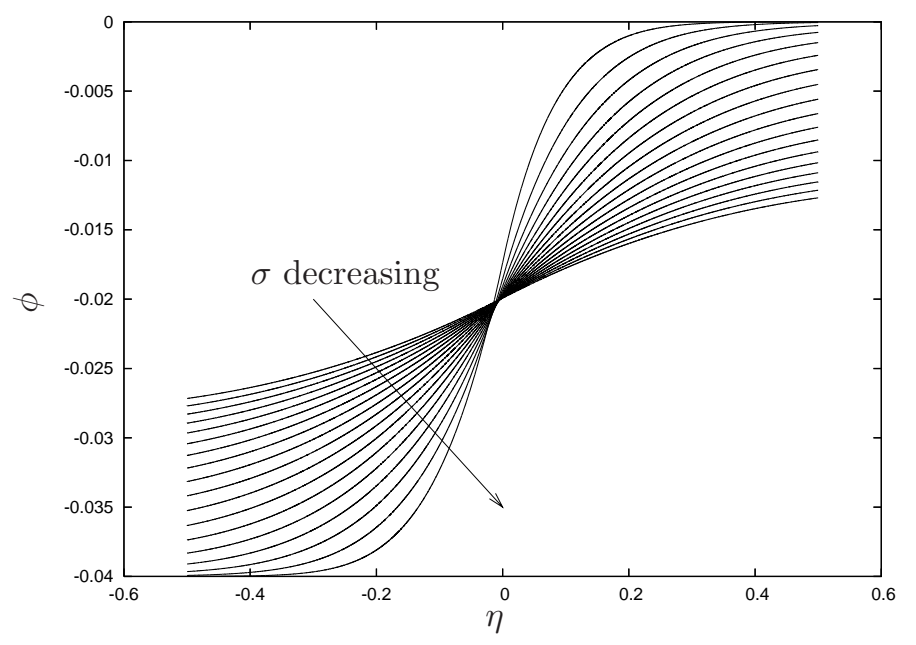

FIG. 4.2. Local $(\tau \rightarrow 0)$ solution of a full-feedback put, $X=1, \lambda=0.1, r=0.04, \sigma=1,0.95$, ..., 0.15 .

4.2. Numerical results - full problem. We now revisit the choice of parameters made in figure 4.1, which led to the aforementioned difficulties. The analysis in the previous subsection points to a discontinuity in the delta at the strike price $(S=X)$ of +1 in the case of a put option. In order to incorporate this into our numerics, an alternative strategy was adopted, based on the Keller (1978) scheme. This modified procedure involved writing (2.10) as a system of two first-order equations namely in $V(S, t)$ and $V_{1}(S, t)=\partial V / \partial S$. The grid was then chosen in such a manner that the strike price $X$ coincided with the $S$ grid. At $S=X$ two values of the option price and its delta were computed, namely $V^{-}$and $V_{1}^{-}$(for $S=X^{-}$) and $V^{+}$and $V_{1}^{+}$(for $S=X^{+}$), such that $V^{-}=V^{+}$and $V_{1}^{+}=V_{1}^{-}+1$. This latter condition effectively built the jump in the delta at the strike price into the numerical scheme. In the time-wise direction, a standard Crank-Nicolson-type scheme was adopted. Calculations performed in this manner provided accurate and highly reliable results, as evidenced in figure 4.3, showing distributions of $V(S, t)-\max (X-S, 0)$, i.e. the difference between the option value and payoff, and as such can be compared directly with the small-time-to-maturity solutions displayed in figure 4.2. Furthermore, figure 4.4 shows the corresponding distributions of the delta, clearly indicating the jump in its value at $S=X$. The computations shown are highly robust (i.e. grid independent), which adds significant credence to the integrity of the results, in particular to the correctness of the jump condition.

There is, however, a further issue relating to the results observed in figure 4.3, namely that this indicates the put option value (close to expiry) is always less then the option payoff. This has implications for the pricing of American options in this framework, because if the European option value is always below the payoff immediately prior to expiry, then the corresponding American option will always be exercised immediately the contract is initiated (at $t=0$ ), i.e. the solution to the American put will be the (trivial) payoff for all time; this could also be regarded as a somewhat undesirable and unrealistic feature of the model.

A corollary to the above remarks is that it can also be seen that the model permits negative values for put options. Whilst in certain extreme option valuations, such as 


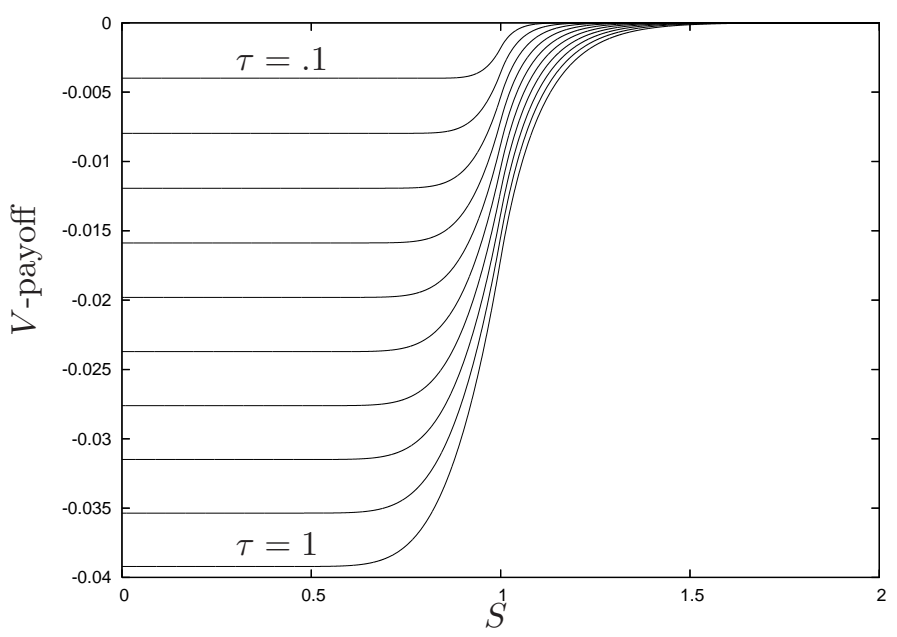

FIG. 4.3. Full feedback put, $X=1, r=0.04, \sigma=0.2, \lambda=0.1$; modified numerical scheme.

those involving storage costs (albeit not part of our model), this may be acceptable, generally this may be regarded as an unwanted facet of the model; indeed, this suggests a breakdown of the no-arbitrage principle. It makes little financial sense to allow negative option values in any model incorporating market frictions, at least under the dynamic hedging (replication) pricing paradigm. For example, in transaction cost models the writer would not re-hedge his portfolio (and hence incur extra transaction costs) at times when he does not need to, provided the option is still perfectly hedged. The same is true for liquidity, the price being modeled is the cost of replicating the option by trading in the underlying. In doing this, we have freedom in our hedging strategy, provided it perfectly replicates the option payoff. Essentially the hedging strategy should never force the hedger into an irrational position. This could be avoided in practice by imposing the condition $V \geq 0$ which effectively creates another free boundary on the PDE at $V=0$ (Bakstein and Howison (2003) call this condition the 'American' constraint).

Figure 4.5 shows results (option value - payoff) for the corresponding call. This clearly reveals that call values not only remain positive, but are also always above the payoff and, hence, indicates that there is no value in early exercise.

It is also of some interest to note that solutions, distinct from the classical BlackScholes solution can be found if we employ the same numerical treatment as outlined above when $\lambda=0$ (even though the problem reduces to a linear one in this case), i.e. we permit discontinuities in the delta of the Black-Scholes option value. A sample result, for a put option, with the same parameters as figure 4.4 are shown in figure 4.6.

Returning now to the other regime outlined in subsection 4.1, i.e. when $1+\frac{8 \lambda \psi}{\sigma^{2} X^{2}}<$ 0 , this turns out to be even more problematic, since here even the $\tau \ll 1$ regime is unclear. It was therefore decided to mount an homotopy type of approach in this regime, specifically by considering a payoff function of the form

$$
V(S, t=T)=\frac{1}{2}\left(X-S+\sqrt{(X-S)^{2}+\rho^{2}}\right)
$$

in conjunction with the full problem (2.10). In this way, it is possible to mimic a 


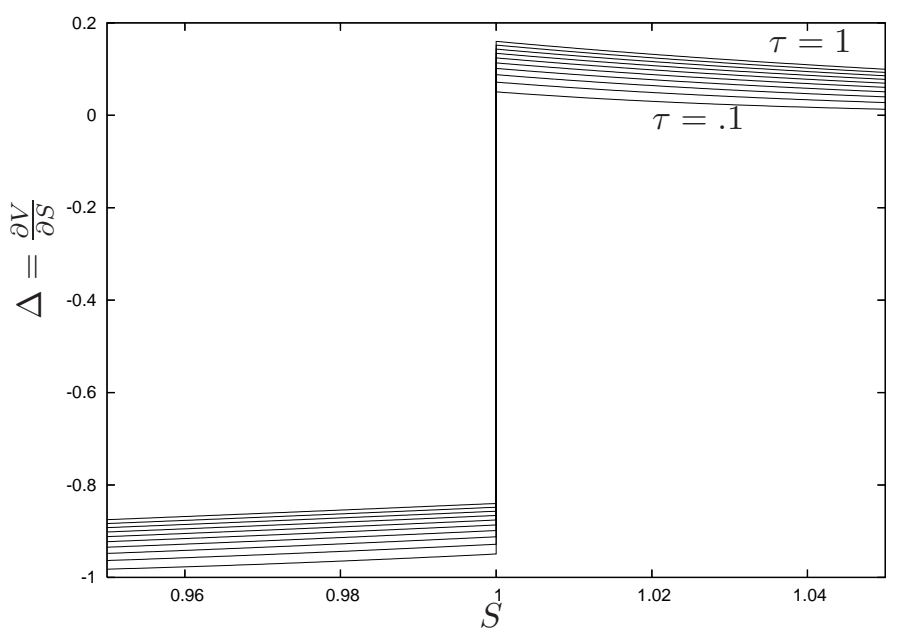

FIG. 4.4. Full feedback put, $X=1, r=0.04, \sigma=0.2, \lambda=0.1$; modified numerical scheme.

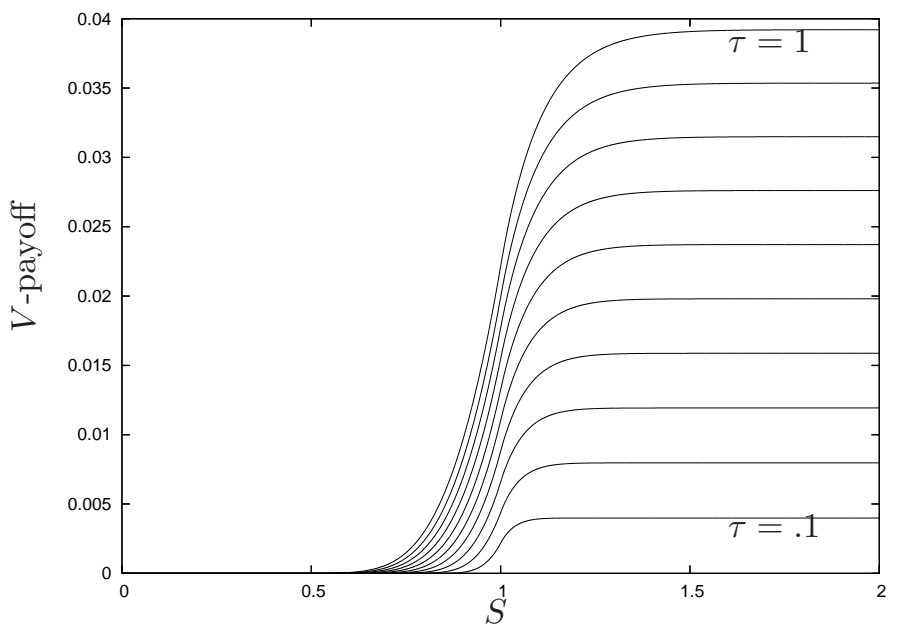

Fig. 4.5. Full feedback call, $X=1, r=0.04, \sigma=0.2, \lambda=0.1$; modified numerical scheme.

standard put payoff as the smoothing parameter $\rho \rightarrow 0$.

In Frey and Stremme (1997) and Frey (1998) this smoothed payoff profile was used to represent an 'idealized' option payoff, which represented a well-diversified portfolio containing a multitude of different payoffs with different strikes which combine to produce a sufficiently smooth payoff to satisfy the smoothness assumptions imposed for existence and uniqueness. Here its use is slightly different, it is merely a mathematical tool to investigate the limit of smoothness. Results corresponding to the parameter choice of figure 4.3, but instead with $\sigma=0.1$ and at a time shortly before expiry $(\tau=0.1)$ and for three choices of $\rho$ are shown in figure 4.7. These results were based on the method employed for figure 4.1, but were tested extensively for numerical grid convergence and found to be numerically consistent on the scale shown.

These calculations strongly indicate that in the limit as $\rho \rightarrow 0$, the solution for 


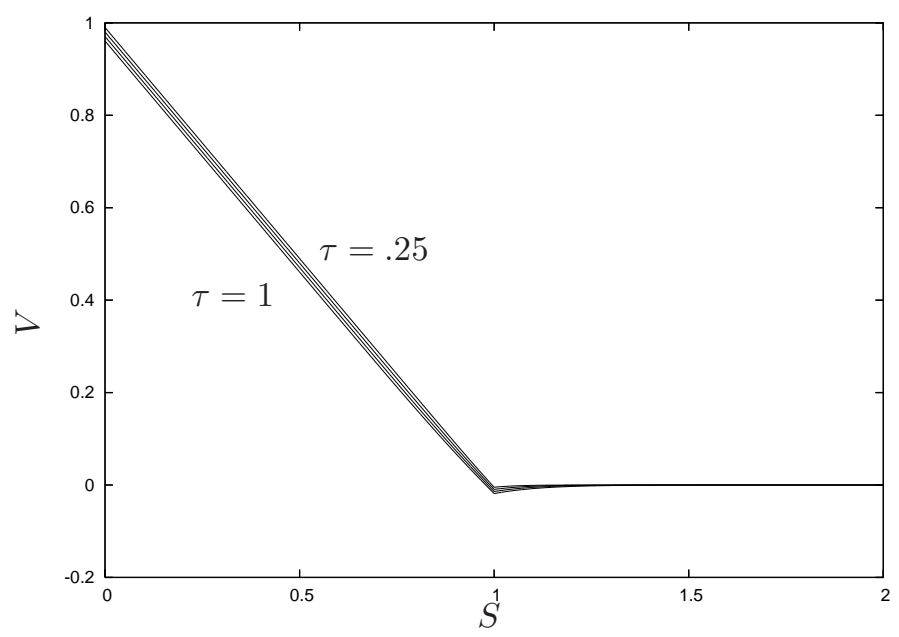

FIG. 4.6. $X=1, r=0.04, \sigma=0.2, \lambda=0$; alternative (non-smooth) Black-Scholes solution.

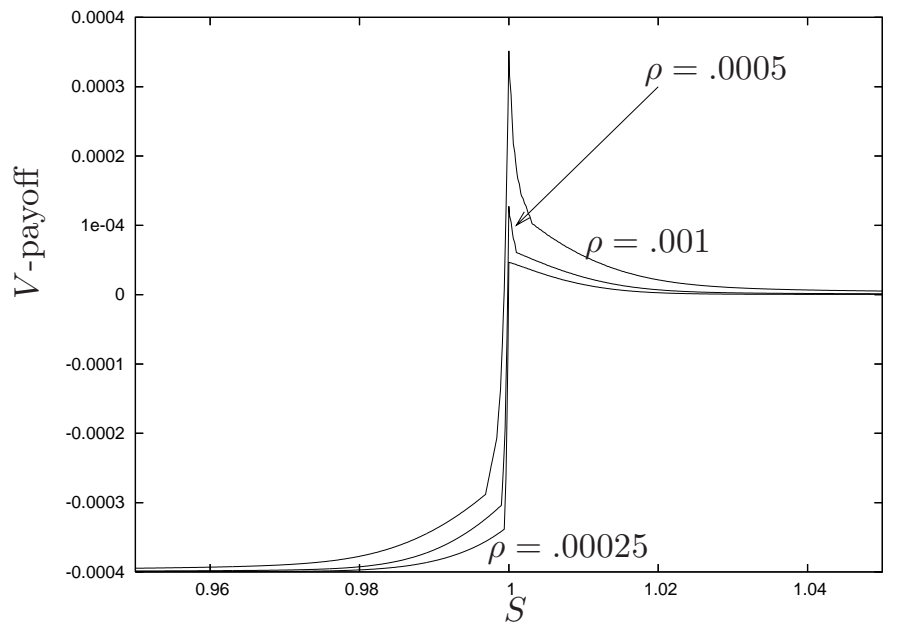

FIG. 4.7. Full feedback put, smoothed payoff, $X=1, r=0.04, \sigma=0.1, \lambda=0.1, \tau=0.1$.

the put takes the trivial form:

$$
V(S, \tau)=\left\{\begin{array}{cc}
0 & \text { for } \quad S>X, \\
X e^{-r \tau}-S & \text { for } \quad S<X
\end{array}\right.
$$

for all time, solutions which do (trivially) satisfy (2.10), and resemble the small volatility results of Widdicks et al. (2005). Note that this form indicates discontinuous option values and also that (4.5) indicates that American options in this regime will always be exercised immediately (at $t=0$ ), for the same reasons expounded earlier for the other regime.

One interpretation of the above results is that the effect of the nonlinearity, for standard (non-smooth) payoff profiles, is to suppress the diffusion term of the equation in regions of non-smoothness, thereby failing to smooth out any discontinuities in the derivative of the payoff profile, as would normally be the case with the (linear) Black- 
Scholes equation.

5. Smoothed payoffs - another breakdown. So far, the difficulties encountered have been attributed to the discontinuous delta, i.e. infinite gamma $\left(\frac{\partial^{2} V}{\partial S^{2}}\right)$, of the payoff profile. If we instead assume smoothness in the payoff, it can be seen that there is the potential for further difficulties to arise due to the vanishing of the denominator in (2.10), i.e. when $V_{S S}=1 / \lambda$. Note that for sufficiently smooth payoff profiles this may never occur, but to illustrate the circumstances under which we should expect such singular behavior, we will once again consider the smoothed payoff profile (4.4), which gives

$$
V_{S S}(S, t=T)=\frac{\rho^{2}}{2\left((S-X)^{2}+\rho^{2}\right)^{\frac{3}{2}}},
$$

which, after equating this to $1 / \lambda$, gives the two (possible) critical locations as

$$
S_{0}=X \pm\left[\left(\frac{\lambda \rho^{2}}{2}\right)^{\frac{2}{3}}-\rho^{2}\right]^{\frac{1}{2}}
$$

which has real solutions if and only if $2 \rho<\lambda$, implying that the denominator does not vanish if this condition is not satisfied. Note that the results for existence and uniqueness of a replicating portfolio provided by Frey (1998) only apply when the denominator is not allowed to vanish (here we impose no such restriction). Hence we have a limit on the smoothness of the payoff profile (parameterized by $\rho$ ) under which the denominator in (2.10) can become zero and so we can expect difficulties with the solution. It should be noted that problems associated with the vanishing of the denominator have been highlighted previously in the literature, but that this regime has deliberately been avoided. For example Sircar and Papanicolaou (1998) set the option value to be the Black-Scholes price a small time $\epsilon$ prior to expiry, where $\epsilon$ is determined to be sufficiently large such that the denominator in the diffusion term is always positive. In addition, Frey and Patie (2002) modified the diffusion term of the equation in an $a d$ hoc manner to prevent the denominator reaching zero. Here we make no such modifications and attempt to fully investigate the nature of these singularities. A solution in their vicinity is sought by again performing a local analysis, about the points $S=S_{0}$, where $V_{S S}\left(S=S_{0}, \tau=0\right)=1 / \lambda$. As $S \rightarrow S_{0}$, $\tau \rightarrow 0$, we then expect a solution of the form

$$
V=V_{0}+\left(S-S_{0}\right) V_{1}+\frac{1}{2 \lambda}\left(S-S_{0}\right)^{2}+\frac{V_{3}}{3 !}\left(S-S_{0}\right)^{3}+\ldots+\frac{2 \sigma^{2} S_{0}^{2} \tau}{\lambda^{3} V_{3}^{2}\left(S-S_{0}\right)^{2}}+\ldots
$$

where $V_{3}=V_{S S S}\left(S_{0}, \tau=0\right)$ and the last term is obtained from a balancing of the diffusion term and the time derivative in (2.10). Effectively, we have assumed a regular expansion from the regularized payoff profile about $S=S_{0}, \tau=0$. The key term is the last, which is clearly singular as $S \rightarrow S_{0}$. However phase-plane analysis (see Glover, 2008) indicates that it is not possible to resolve this singular behavior, even using small-scale analysis, suggesting that, despite applying a smoothed payoff profile, singularities have been 'induced' into the solution for $\tau>0$. The corollary to this is, therefore, that there is insufficient financial modeling in (2.10) to prevent such behavior, for standard puts and calls at least, indicating (another) failure in the underlying modeling. Finally, it should be pointed out that strictly the parameter 
values taken in figure 4.7 are in the range $\rho<\frac{\lambda}{2}$, as described above. However, there is a further subtlety as $\rho \rightarrow 0$ (which we do not explore), insofar as in this limit yet further asymptotic analysis is applicable, involving another small parameter, namely $\rho$ itself. Note too that as $\rho \rightarrow 0$, the two values of $S_{0}$ will coincide and in this limit the problems associated with the vanishing of the denominator are in some ways mediated by the problems of the infinite gamma. Figure 4.7 is still useful, however, in guiding the asymptotics described above.

6. Perpetual Options. Although section 4.2 has indicated that the full-feedback model with early exercise leads to what amounts to a trivial problem for puts, the question that naturally arises (given the results of the previous section) is what of other payoff conditions, in particular those which do not have discontinuous deltas (and assuming the difficulties raised in section 5 can be bypassed). The next set of results (obtained using a straightforward PSOR scheme), shown in figure 6.1, correspond to a calculation obtained taking the smoothed payoff condition (4.4), as described above. This set of results (for an American-style put option) corresponds to the payoff condition with $\rho=0.15$ (together with $X=1, r=0.04, \sigma=0.2, \lambda=0.25$ ), i.e. in this parameter regime the denominator does not vanish. To be consistent with the final payoff conditions, the early-exercise condition was imposed by taking

$$
V=\max \left[\frac{1}{2}\left(X-S+\sqrt{(X-S)^{2}+\rho^{2}}\right), V_{\mathrm{PDE}}\right],
$$

at all $S$ and $t$ at each iteration, where $V_{\mathrm{PDE}}$ is the solution to (2.10). The computation was permitted to continue until a near steady state had been attained (i.e. the asymptote to a perpetual valuation). Figure 6.1 clearly indicates that the computation could be extended, unabated, for long maturities. This does emphasize, of course, that much of the difficulty reported above with standard payoff conditions is associated with the denominator in (2.10) (which will certainly be the case for standard payoff functions on account of the discontinuous deltas) Furthermore, applying the above numerical treatment to the regime in which the denominator is expected to vanish proved challenging and no consistent results could be obtained.

Given that long-term solutions to (2.10) (with early exercise) can exist, it is of some interest to investigate the behavior of this system with the time variation omitted, i.e.

$$
\frac{1}{2} \sigma^{2} S^{2} \frac{V_{S S}}{\left(1-\lambda V_{S S}\right)^{2}}+r S V_{S}-r V=0,
$$

subject to (the standard early-exercise put conditions) $V \rightarrow 0$ as $S \rightarrow \infty$, and $V=$ $X-S, \frac{d V}{d S}=-1$ on the free boundary $S=S_{f}$. This system was solved using a straightforward Runge-Kutta algorithm, which performed an iteration procedure to evaluate $S_{f}$. Results, based on (6.2) are shown in figure 6.2 for a range of values of the parameter $\lambda$, with $X=1, \sigma=0.2, r=0.04$. The location of the free boundary is also clearly marked, and thus reveals yet another interesting feature, namely the approach of the free boundary towards $S=0$. For $\lambda \gtrsim 1.1$, for the choice of parameters taken above, it would appear that no perpetual solution of this form exists (the free boundary reaches $S=0$ at $\lambda \approx 1.1$ ).

7. Conclusions. We have investigated models which have been proposed to incorporate illiquidity of the underlying into the classical Black-Scholes-Merton framework. A feature common to a number of these models is that the overall dispersion 


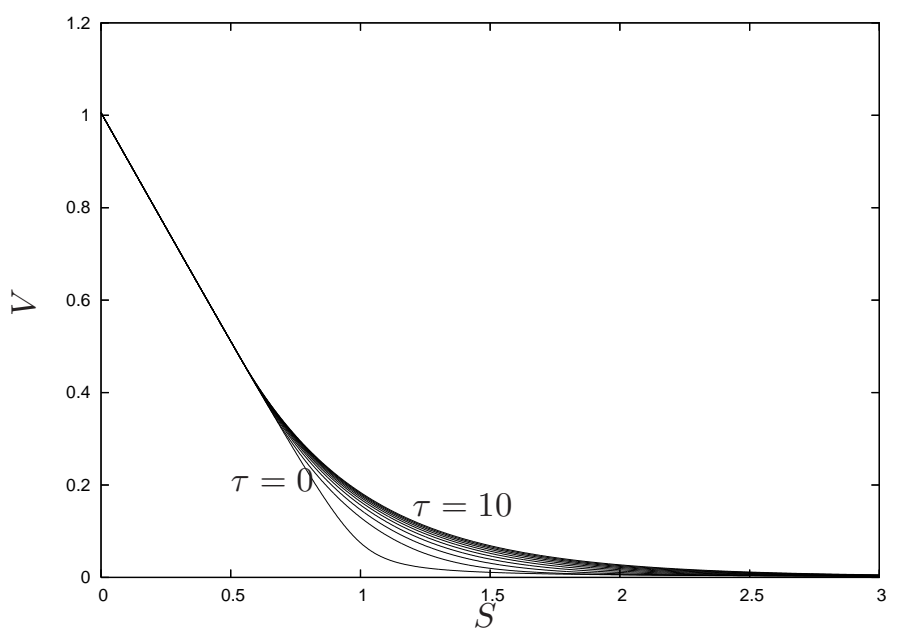

FIG. 6.1. Full feedback American put, $X=1, r=0.04, \sigma=0.2, \lambda=0.25, \rho=0.15$ (smoothed payoff), $\tau=0,1, \ldots, 10$.

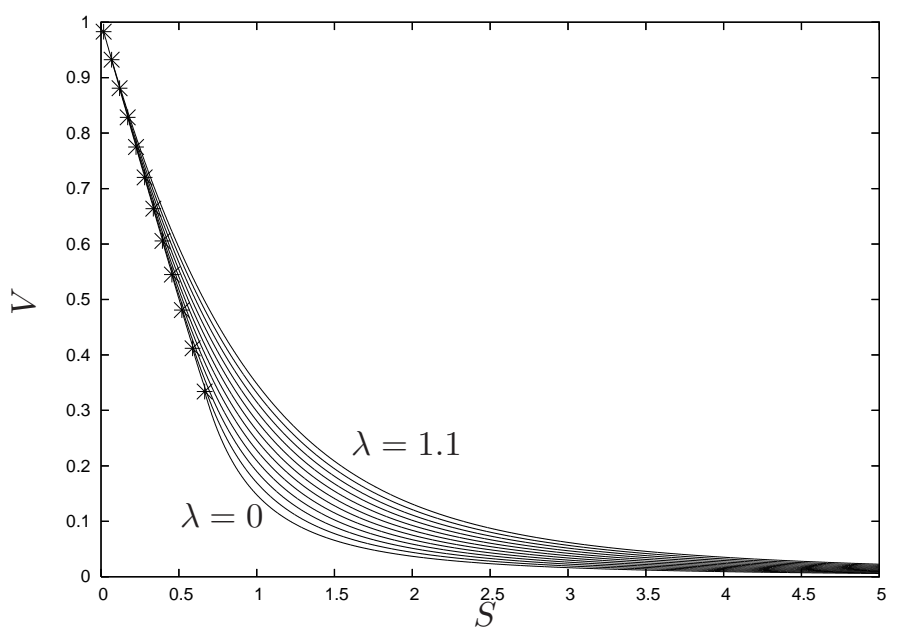

Fig. 6.2. Perpetual full-feedback American put, $X=1, r=0.04, \sigma=0.2, \lambda=$ $0,0.1,0.2, \ldots, 1.1$; free boundary location as indicated.

term, involving the option gamma, diminishes in magnitude as the gamma increases in magnitude (as indeed it must as standard payoff conditions are approached). The upshot of this is that models of this general class cannot exhibit fully differentiable solutions at times prior to expiry; instead, we must allow solutions with discontinuous deltas. This is clearly a somewhat undesirable feature, which is exacerbated by the possibility of negative option values for puts. These features can be gleaned from a detailed asymptotic analysis, valid close to expiry. Indeed, invariably these solution features lead to completely spurious solutions if standard numerical procedures are adopted. However, the insight obtained about the governing PDE from this analysis also gives guidance on how to tackle these problems numerically at times away from expiry (the full problem), incorporating the appropriate discontinuities into the nu- 
merical scheme. Allied to this, the vanishing of the denominator in (2.10) can also be a serious issue. It is concluded that there is insufficient financial modeling in equation (2.10) to describe the true price dynamics in such situations.

It is clear that the period close to expiry is the most critical for option-pricing models and any model that successfully treats this regime should also successfully replicate the option value dynamics for all time. The approach detailed in this paper should give guidance for the development of models incorporating illiquidity and permanent price impact without the undesirable features observed in a number of the existing models. Indeed, the local analysis methods used throughout this paper are quite generically applicable, and have been employed on other models recently by Glover (2008). Several models in the past have circumvented these difficulties close to expiry but generally using ad hoc, rather than intuitively justifiable arguments. The hope is that the analysis presented in this paper will help in this respect.

\section{References.}

Avellaneda, M., Levy, A., and Paras, A. (1995). Pricing and hedging derivative securities in markets with uncertain volatilities. Applied Mathematical Finance, 2:73-88.

Bakstein, D. and Howison, S. (2003). A non-arbitrage liquidity model with observable parameters. Working paper, Mathematical Institute, Oxford University. Available at: http://eprints.maths.ox.ac.uk/53/.

Black, F. and Scholes, M. (1973). The pricing of options and corporate liabilities. Journal of Political Economy, 81:637-659.

Cetin, U., Jarrow, R. A., and Protter, P. (2004). Liquidity risk and arbitrage pricing theory. Finance and Stochastics, 8:311-341.

Cetin, U., Jarrow, R. A., Protter, P., and Warachka, M. (2006). Pricing options in an extended Black Scholes economy with illiquidity: theory and empirical evidence. Review of Financial Studies, 19:493-529.

Cetin, U. and Rogers, L. C. G. (2007). Modeling liquidity effects in discrete time. Mathematical Finance, 17:15-29.

Duffie, D. (1996). Dynamic Asset Pricing Theory, 2nd edition. Princeton University Press.

Frey, R. (1998). Perfect option replication for a large trader. Finance and Stochastics, $2: 115-142$.

Frey, R. (2000). Market illiquidity as a source of model risk in dynamic hedging. Model Risk, pages 125-136.

Frey, R. and Patie, P. (2002). Risk management for derivatives in illiquid markets: A simulation study. In: $K$ Sandmann and $P$ Schönbucher (Eds.), Advances in Finance and Stochastics, Springer, pages 137-159.

Frey, R. and Stremme, A. (1997). Market volatility and feedback effects from dynamic hedging. Mathematical Finance, 7:351-374.

Glover, K. J. (2008). The analysis of PDEs arising in nonlinear and non-standard option pricing. Ph.D. thesis, University of Manchester, UK.

Jarrow, R. A. (1994). Derivative securities markets, market manipulation and option pricing theory. Journal of Financial Quantitative Analysis, 29:241-261.

Keller, H. (1978). Numerical methods in boundary-layer theory. Ann. Rev. Fluid Mech., 10:417 - 433 .

Kuske, R. A. and Keller, J. B. (1998). Optimal exercise boundary for an american put option. Applied Mathematical Finance, 5:107-116.

Lipton, A. (2001). Mathematical Methods for Foreign Exchange. World Scientific. 
Liu, H. and Yong, J. (2005). Option pricing with an illiquid underlying asset market. Journal of Economic Dynamics \& Control, 29:2125-2156.

Lyukov, A. (2004). Option pricing with feedback effects. International Journal of Theoretical and Applied Finance., 7:757-768.

Madhavan, A. (2000). Market microstructure: A survey. Journal of Financial Markets., 3:205-258.

Merton, R. C. (1973). Theory of rational option pricing. Bell J. Econ. Manag. Sci., 4:141-183.

Platen, E. and Schweizer, M. (1998). On feedback effects from hedging derivatives. Mathematical Finance, 8:67-84.

Schönbucher, P. and Wilmott, P. (2000). The feedback effect of hedging in illiquid markets. SIAM Journal of Applied Mathematics, 61:232-272.

Sircar, K. and Papanicolaou, G. (1998). General Black-Scholes models accounting for increased market volatility from hedging strategies. Applied Mathematical Finance, $5: 45-82$.

Whalley, A. E. and Wilmott, P. (1993). Counting the costs. Risk Magazine, 6:59-66.

Widdicks, M. (2002). Examination, extension and creation of methods for pricing options with early exercise features. Ph.D. thesis, University of Manchester, UK.

Widdicks, M., Duck, P. W., Andricopoulos, A. D., and Newton, D. P. (2005). The Black-Scholes equation revisited: Asymptotic expansions and singular perturbation. Mathematical Finance, 15:373-391.

Wilmott, P., Howison, S., and Dewynne, J. (1995). The Mathematics of Financial Derivatives. Cambridge University Press. 